Western University

Scholarship@Western

Brain and Mind Institute Researchers'

Publications

Brain and Mind Institute

10-1-1997

Triple dissociation of anterior cingulate, posterior cingulate, and medial frontal cortices on visual discrimination tasks using a touchscreen testing procedure for the rat.

\author{
T J Bussey \\ J L Muir \\ B J Everitt \\ T W Robbins
}

Follow this and additional works at: https://ir.lib.uwo.ca/brainpub

Part of the Neurosciences Commons, and the Psychology Commons

Citation of this paper:

Bussey, T J; Muir, J L; Everitt, B J; and Robbins, T W, "Triple dissociation of anterior cingulate, posterior cingulate, and medial frontal cortices on visual discrimination tasks using a touchscreen testing procedure for the rat." (1997). Brain and Mind Institute Researchers' Publications. 84.

https://ir.lib.uwo.ca/brainpub/84 


\title{
Triple Dissociation of Anterior Cingulate, Posterior Cingulate, and Medial Frontal Cortices on Visual Discrimination Tasks Using a Touchscreen Testing Procedure for the Rat
}

\author{
Timothy J. Bussey, Janice L. Muir, Barry J. Everitt, and Trevor W. Robbins \\ University of Cambridge
}

\begin{abstract}
Four experiments examined effects of quinolinic acid-induced lesions of the anterior cingulate, posterior cingulate, and medial frontal cortices on tests of visual discrimination learning, using a new "touchscreen" testing method for rats. Anterior cingulate cortex lesions impaired acquisition of an 8-pair concurrent discrimination task, whereas posterior cingulate cortex lesions facilitated learning but selectively impaired the late stages of acquisition of a visuospatial conditional discrimination. Medial frontal cortex lesions selectively impaired reversal learning when stimuli were difficult to discriminate; lesions of anterior and posterior cingulate cortex had no effect. These results suggest roles for the anterior cingulate, posterior cingulate, and medial frontal cortex in stimulus-reward learning, stimulus-response learning or response generation, and attention during learning, respectively.
\end{abstract}

Devinsky, Morrell, and Vogt (1995) and Vogt, Finch, and Olson (1992) provided evidence that in human and infrahuman primates, different functions are carried out by anatomically distinct subregions of the cingulate cortex. Similar anatomical heterogeneity exists in the cingulate cortex of the rat (e.g., Kolb, 1984, 1990; Lopez da Silva, Witter, Boeijinga, \& Lohman, 1990; Vogt, 1985), suggesting that this species, with its many advantages as an experimental subject, may be particularly useful in the systematic investigation of cingulate cortex function. Indeed, functional dissociations of subregions of the cingulate cortex in rats have been described already. For example, R. J. Sutherland, Whishaw, and Kolb (1988) reported that posterior, but not anterior, cingulate cortex lesions impair spatial learning in a water maze. Muir, Everitt, and Robbins (1996), using a continuous performance test of visual attention (the "fivechoice serial reaction time task"), showed that although medial frontal cortex lesions led to impaired choice accuracy and slower response times, anterior cingulate cortex lesions produced an increase only in premature "anticipatory responses." Furthermore, anterior, but not posterior, cingulate cortex lesions have been reported to impair serial reversal learning in a $T$ maze, whereas posterior cingulate cortex lesions disrupted only the initial discrimination and first reversal (Meunier, Jaffard, \& Destrade, 1991). Finally, particularly compelling data have been provided by Gabriel (1993) and his colleagues showing that although anterior

Timothy J. Bussey, Janice L. Muir, Barry J. Everitt, and Trevor W. Robbins, Department of Experimental Psychology, University of Cambridge, Cambridge, England.

This research was partly supported by a Wellcome Trust Programme grant and partly by a Commonwealth Scholarship.

Correspondence concerning this article should be addressed to Trevor W. Robbins, Department of Experimental Psychology, University of Cambridge, Downing Street, Cambridge, CB2 3EB England. Electronic mail may be sent via Internet to twr2@cus.cam. ac.uk. cingulate cortex lesions disrupt the acquisition of active avoidance during the early stages of learning, posterior cingulate cortex lesions impair only the late stages of acquisition of this task. Unit-recording studies have shown that neurons develop preferential firing to the CS+ (which predicts shock) compared with the CS- (which does not) in this paradigm and that this discriminative neuronal activity develops early in learning in the anterior cingulate cortex and only late in learning in the posterior cingulate cortex. Activity in the prefrontal cortex develops earlier than the activity observed in either of these areas (for a review, see Gabriel, 1993).

Our research was concerned with three anatomically distinct cortical regions: the medial frontal cortex, the anterior cingulate cortex, and the posterior cingulate cortex. The medial frontal cortex includes Zilles's (1985) area Cg3 as well as the overlying $\mathrm{Cgl}$ that lies rostral to the genu of the corpus callosum. This area projects to the nucleus accumbens (Brog, Salyapongse, Deutch, \& Zahm, 1993; Kolb, 1984; McGeorge \& Faull, 1989), the mediodorsal nucleus of the thalamus (primarily the medial portion; Uylings \& van Eden, 1990), and the amygdala (Ray \& Price, 1992; Sesack, Deutch, Roth, \& Bunney, 1989). We refer to this region as the "medial frontal cortex" to preserve consistency with the literature and because it is a matter of debate whether this area should be regarded as cingulate cortex. The anterior cingulate cortex corresponds to Zilles's areas $\mathrm{Cg} 1$ and $\mathrm{Cg} 2$, caudal to the genu of the corpus callosum, and has connections with the mediodorsal caudate nucleus (Groenewegen, Berendse, Wolters, \& Lohman, 1990; McGeorge \& Faull, 1989), mediodorsal nucleus of the thalamus (primarily the lateral portion; Uylings \& van Eden, 1990), and the amygdala (Divac \& Diemer, 1980; Sripanidkulchai, Sripanidkulchai, \& Wyss, 1984). The posterior cingulate cortex corresponds to Zilles's retrosplenial agranular cortex (RSA) and retrosplenial granular cortex (RSG), rostral to the splenium of the corpus callosum. Connections 
with this area include the mediodorsal caudate nucleus (McGeorge \& Faull, 1989), anterior thalamus, and hippocampus (Lopez da Silva et al., 1990; Vogt, 1985). Because we did not investigate cingulate areas caudal to the splenium of the corpus callosum, we adopted the term posterior cingulate cortex to describe this region rather than the often-used term retrosplenial cortex.

In the current research we lesioned selectively these areas to investigate the possibility that these anatomically dissociable areas also are functionally distinct and to provide data that may help to elucidate the specific functions of each of these areas. In previous studies, cingulate cortex lesions have often encompassed two or more of these regions and have used aspirative, radio-frequency, or electrolytic lesions. In the current research, we made excitotoxic lesions, which should minimize damage to fibers of passage in these areas. This is particularly important in light of a recent report that damage to the cingulum bundle-a fiber tract passing along the entire length of the cingulate cortex - can by itself produce significant behavioral impairments (Neave, Nagle, Sahgal, \& Aggleton, 1996).

To compare in as controlled a manner as possible the effects of these lesions on various learning tasks, we conducted all experiments in the same automated apparatus using a new "touchscreen" testing method that allows the presentation of computer-graphic stimuli to rats (Bussey et al., 1994). Various tasks may be implemented in this apparatus, in which rats indicate choices by making nose pokes directly toward the selected stimuli. Animals with quinolinic acid-induced lesions of the anterior cingulate, posterior cingulate, and medial frontal cortices were tested in the acquisition of the following tasks: visuospatial conditional discrimination, eight-pair concurrent discrimination, and one-pair discrimination and reversal.

In the visuospatial conditional discrimination task, the animals must learn a rule of the type, "If Stimulus A, then go left and if Stimulus B, then go right." Tasks of this type have been described as requiring "procedural" memory for stimulus-response (S-R) "habits" (Dudchenko \& Sarter, 1991). We were particularly interested in examining the effects of cingulate cortex lesions on this task following our investigations using an analogous task carried out in an operant chamber, in which animals had to learn a rule of the type, "If lights flash fast, press the right lever and if slow, press left." It was found that whereas lesions of the posterior cingulate cortex impaired acquisition of this task selectively during the late stages of task acquisition, lesions of the anterior cingulate cortex facilitated task acquisition selectively during the early stages of learning (Bussey, Muir, Everitt, \& Robbins, 1996). A replication of this result in the touchscreen apparatus using much different stimuli, different response requirements, and different systems of reinforcement would provide strong evidence that these results were not attributable to nonspecific factors but instead were related to the cognitive demands of this task.

In the eight-pair concurrent discrimination task, eight pairs of stimuli are presented concurrently during a session and the rat must learn which stimulus in each pair is associated with a reward. Zola-Morgan and Squire (1985) suggested that concurrent discrimination learning tasks are sensitive tests of amnesia in humans because of a high memory load for stimulus-reward associations. We used this task because it has been hypothesized that the anterior cingulate cortex may have a role in stimulus-reward learning (Bussey et al., 1996).

Although humans with amnesia and monkeys with damage to the temporal lobes are impaired on eight-pair concurrent discrimination learning, they are unimpaired on simple one-pair discriminations. By including a one-pair discrimination in our research, we were able to assess whether any effects observed on the eight-pair concurrent discrimination task were attributable to nonspecific factors.

In addition, we examined rats' performance on reversal learning - in which the previously reinforced stimulus becomes nonreinforced and vice versa- of this one-pair discrimination. This was done specifically to examine the role of the medial frontal cortex of the rat in nonspatial reversal learning. We were particularly interested in investigating the locus in the rat of the perseverative deficits reported after frontal lesions in monkeys (e.g., Dias, Robbins, \& Roberts, 1996; Jones \& Mishkin, 1972) and the possible contribution of perseveration to nonspatial reversal learning deficits in the rat (e.g., Becker \& Olton, 1980). Finally, we tested animals on a one-pair discrimination and reversal using easily discriminable stimuli to assess the effects of altering the perceptual demands of this task.

\section{General Method}

\section{Subjects}

The subjects were 228 male Lister hooded rats (Olac, Bicester, England), weighing $320-350 \mathrm{~g}$ before behavioral testing, housed in pairs in a temperature-controlled room (minimum $22^{\circ} \mathrm{C}$ ) under 12:12-hr light-dark conditions. They were provided with free access to water and were maintained throughout the experiment at $90 \%$ of their free-feeding weight using a restricted feeding regimen.

\section{Surgical Procedures}

The rats received administration of an anaesthetic $(0.3 \mathrm{ml} / 100 \mathrm{~g}$ ip) with the following composition: $81 \mathrm{ml}$ of Nembutal (Rhone Merieux Ltd., Harlow, England) containing 10\% vol/vol alcohol plus $20 \% \mathrm{vol} / \mathrm{vol}$ propylene glycol, $21.25 \mathrm{~g}$ chloral hydrate, $10.63 \mathrm{~g}$ $\mathrm{Mg}_{2} \mathrm{SO}_{4}, 198 \mathrm{ml}$ of propane-1,2-diol, and $50 \mathrm{ml}$ absolute alcohol and brought up to $500 \mathrm{ml}$ with distilled water. They were placed in a Kopf stereotaxic frame fitted with atraumatic ear bars. Quinolinic acid (Sigma, Poole, England) was dissolved in $0.1 \mathrm{~mol}$ phosphate buffer ( $\mathrm{pH}=7.2-7.4$ ) to a concentration of $0.09 \mathrm{~mol}$. A volume of $0.5 \mu$ l of this solution was infused through a 30-ga cannula attached to a 10- $\mu$ l microsyringe (SGE, Baton Rouge, LA). Bilateral lesions of the anterior cingulate cortex (ANT group) were made using the following injection coordinates (Paxinos \& Watson, 1986): anteroposterior $(\mathrm{AP})=+0.8$, mediolateral $(\mathrm{ML})= \pm 0.5$, and dorsoventral $(\mathrm{DV})=-3.0$ and $-2.0 ; \mathrm{AP}=+0.2, \mathrm{ML}= \pm 0.5$, and $\mathrm{DV}=$ -2.5 and -2.0 ; and $\mathrm{AP}=-0.4, \mathrm{ML}= \pm 0.5$, and $\mathrm{DV}=-2.0$ and -1.5 (incisor bar set at $2.3 \mathrm{~mm}$ below the interaural line). Bilateral posterior cingulate cortex lesions (POS group) were made in the same manner at the following sites: $\mathrm{AP}=-3.3, \mathrm{ML}= \pm 0.5$, and $\mathrm{DV}=-1.2 ; \mathrm{AP}=-4.0, \mathrm{ML}= \pm 0.5$, and $\mathrm{DV}=-1.2$; and $\mathrm{AP}=$ $-5.0, \mathrm{ML}= \pm 0.5$, and $\mathrm{DV}=-2.0$. Coordinates for the medial 
frontal lesion (MF group) were $\mathrm{AP}=+2.6, \mathrm{ML}= \pm 0.5$, and $\mathrm{DV}=-1.5 ; \mathrm{AP}=+3.3, \mathrm{ML}= \pm 0.5$, and $\mathrm{DV}=-3.0$ and -1.5 ; and $\mathrm{AP}=+3.8, \mathrm{ML}= \pm 0.5$, and $\mathrm{DV}=-1.5$. After each infusion, the injection cannula was left in place for another $2 \mathrm{~min}$. Sham lesions of the anterior cingulate cortex, posterior cingulate cortex, and medial frontal cortex were made using the coordinates and procedures just outlined, except that no infusion was made after cannula insertion.

\section{Histology}

At the conclusion of behavioral testing, the animals were perfused transcardially with $0.9 \%$ saline followed by $10 \%$ formol saline. After dehydration by immersion in $30 \%$ sucrose, the brains were sectioned on a freezing microtome at $60-\mu \mathrm{m}$ thickness. Every fourth section through the region of the lesions was collected, mounted on glass slides, and stained with Cresyl Violet. These sections were used to verify lesion placement and to assess the extent of lesion-induced neuronal loss.

\section{Apparatus}

A preliminary description of the apparatus and testing methods is provided by Bussey et al. (1994). The apparatus, shown in Figure 1 (built in the Department of Experimental Psychology, University of Cambridge), consisted of a testing chamber and video display unit
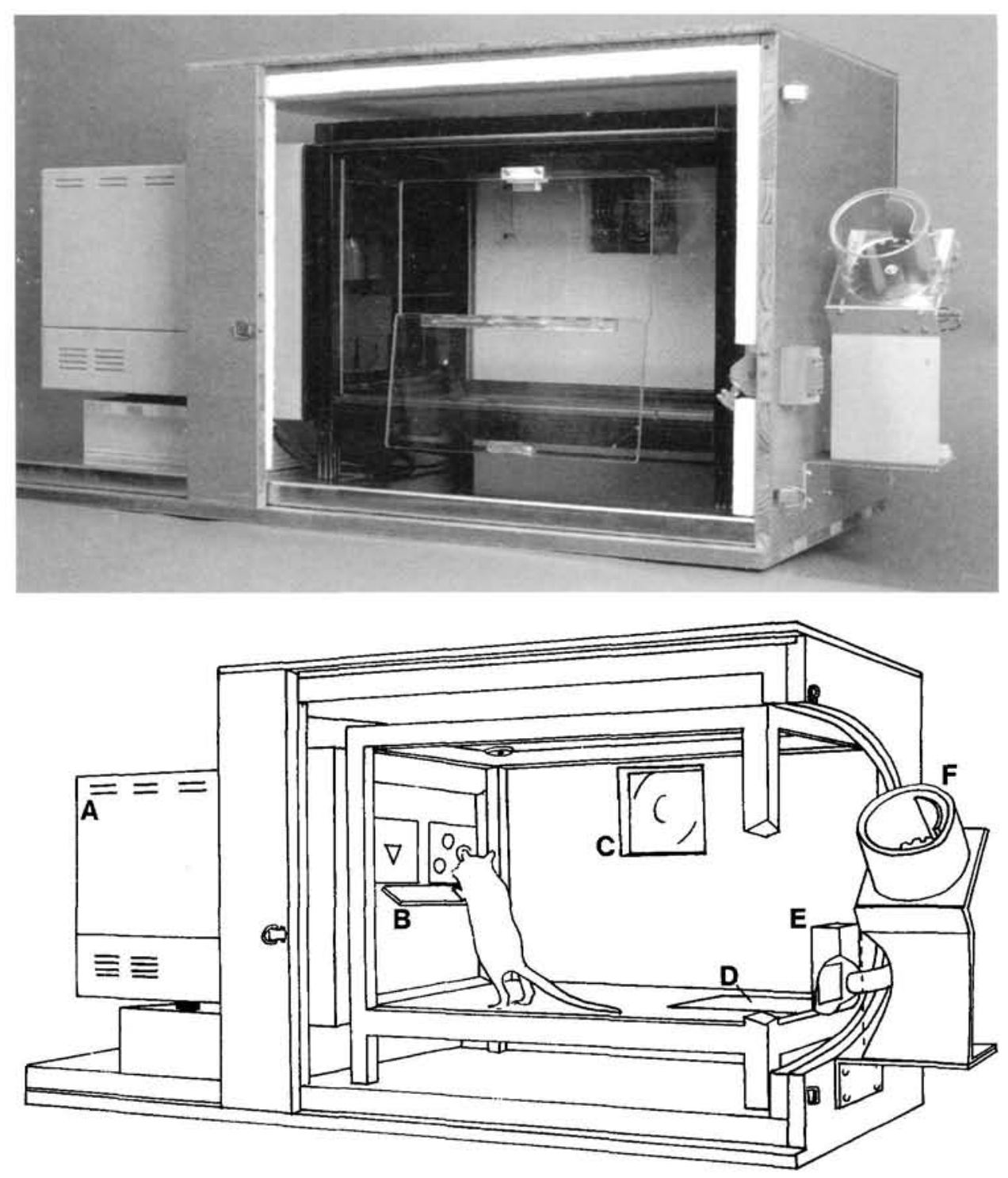

Figure 1. Photograph and line drawing of the apparatus. $\mathrm{A}=$ video display unit; $\mathrm{B}=$ Perspex "mask" with response windows and "shelf"; $\mathrm{C}=$ fan; $\mathrm{D}=$ pressure-sensitive floor panel; $\mathrm{E}=$ magazine; $\mathrm{F}=$ pellet dispenser. Illustration by Brian Fromant, Department of Experimental Psychology, Cambridge. From "A Novel Automated Touchscreen Procedure for Assessing Learning in the Rat Using Computer Graphic Stimuli," by T. J. Bussey, J. L. Muir, and T. W. Robbins, 1994, Neuroscience Research Communications, 15, p. 105. Copyright 1994 by John Wiley and Sons, Ltd. Reprinted with permission. 
(VDU) housed within a wooden sound-attenuating box, fitted with a fan for ventilation and masking of extraneous noise. The inner chamber measured $48 \times 30 \times 30 \mathrm{~cm}$ and consisted of a metal frame, clear Perspex walls, and an aluminum floor. A 3-W houselight and a tone generator were attached to the ceiling of the chamber. Located centrally on the wall at the rear of the chamber was a food magazine attached to a pellet dispenser (Campden Instruments, Loughborough, England) situated outside the soundattenuating box. The magazine could be illuminated by a standard 3-W bulb. Animals gained access to the magazine via a hinged Perspex panel monitored by a microswitch. A pressure-sensitive area of floor measuring $14 \times 10 \mathrm{~cm}$ and located directly in front of the food magazine was attached to a microswitch to detect the presence of the rat when in this area of the testing chamber. The VDU, on which the stimuli were presented, was located at the other end of the chamber. Surrounding the VDU was a "touchscreen" attachment (Touch-tech 501, Microvitec, Bradford, England), which is an array of horizontally and vertically placed photocells that detect the location of pokes to the VDU screen. Because the array of photocells was located approximately $1 \mathrm{~cm}$ from the VDU screen, the rats were not required to make contact with the screen, only to come close to touching it. A black Perspex "mask" was attached to the face of the VDU approximately $2 \mathrm{~cm}$ from the surface of the display. This mask served to block access to the VDU display except through response windows measuring $6 \times 8 \mathrm{~cm}$. A shelf extending $7 \mathrm{~cm}$ from the surface of the Perspex mask was positioned just beneath the response windows, approximately 15 $\mathrm{cm}$ from the floor of the chamber. The shelf was supported by springs to prevent attempts by animals to climb onto it. The combined effect of the response windows and the shelf was to force the animal to stop, rear up, and stretch toward the stimuli with a head-on approach, thus facilitating the rats' attention to the stimuli. The stimuli used in the tasks consisted of colored shapes, lines, and patterns (see Figure 2). The apparatus was controlled and monitored by a BBC Master series microcomputer using programs written by one of us in BBC BASIC.

\section{General Behavioral Procedures}

In each of the three behavioral tasks, each trial began with the presentation of the stimuli, which was contingent on the animal being located on the rear floor panel after a 5-s intertrial interval. The rat was then required to approach the VDU and select a stimulus by responding to it directly via a nose poke. Correct responses were followed by the disappearance of the stimuli and the presentation of a 1-s, 4-kHz tone, concomitant with illumination of the magazine light and delivery of a sucrose pellet (45-mg dustless pellets; Bioserve, Inc., Frenchtown, NJ) into the magazine. Incorrect responses resulted in the disappearance of the stimuli and the houselight being extinguished for a time-out period of $5 \mathrm{~s}$. In the event that a rat developed a spatial response bias (defined as more than $70 \%$ responses to the left or right response window within a session), a correction procedure was implemented such that after an incorrect response, the stimulus configuration for that trial was re-presented until the rat responded correctly. For the eight-pair concurrent discrimination task, the number of trials per session was increased appropriately during correction sessions such that each of the eight stimulus pairs was presented at least 15 times. Each rat typically required only one or two correction sessions.

\section{Pretraining}

Pretraining began 10 days after surgery and consisted of four sessions. During the first 30 -min session, the magazine was filled with sucrose pellets that held open the magazine panel, allowing the animal unrestricted access to pellets. As the rat ate the pellets, the magazine panel closed, thus requiring the animal to push open the panel to gain access to the remaining pellets. On completion of a session in which all of pellets were consumed (usually the first one), animals were given two 30 -min sessions in which pellets were delivered under a variable interval 40 -s schedule together with illumination of the magazine light and presentation of the tone. When rats were reliably retrieving pellets from the magazine following delivery (resulting in a minimum of 60 pellets per session), they were trained to respond to the VDU display. During each of 50 trials, a large yellow square was randomly presented in one of the response windows. The square remained on the screen until the rat responded to it, after which the rat was rewarded with magazine light, tone, and sucrose pellet. Once a rat was able to obtain 50 reinforcements within $20 \mathrm{~min}$, it was moved on to a particular task.
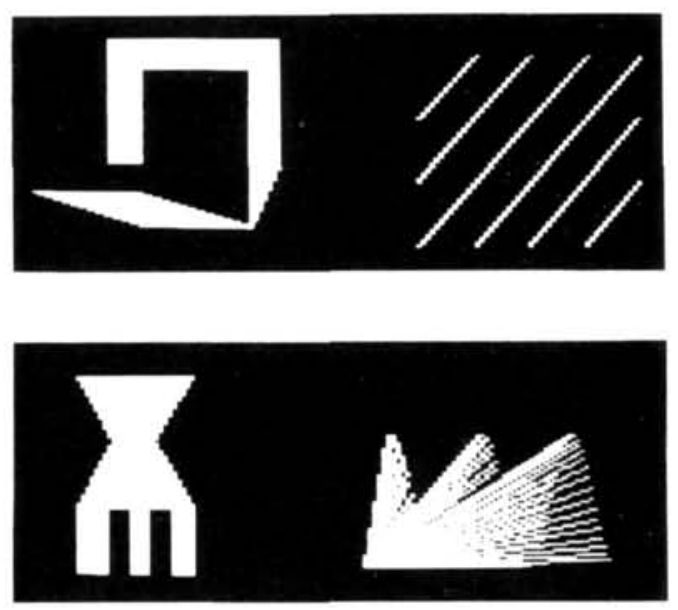

Figure 2. Representative colored shape, line, and pattern stimuli. From "A Novel Automated Touchscreen Procedure for Assessing Learning in the Rat Using Computer Graphic Stimuli," by T. J. Bussey, J. L. Muir, and T. W. Robbins, 1994, Neuroscience Research Communications, 15, p. 106. Copyright 1994 by John Wiley and Sons, Ltd. Reprinted with permission. 


\section{Measures}

Four measures were recorded during acquisition of a given task: (a) number of errors committed; (b) percentage of bias, which was the number of responses either to the right or left response window depending on a particular animal's bias during a session, expressed as a percentage of total trials for that session; (c) average choice latency, which was the time from the onset of the choice stimuli to the time the rat made a nose poke to one of the choice stimuli; and (d) average magazine latency, which was the time from a correct nose poke to the time the rat entered the magazine to collect reward.

\section{Data Analysis}

Data for each variable were subjected to an analysis of variance (ANOVA) using the CLR ANOVA Version 2 (Clear Lake Research, Houston, TX) statistical package. Further post hoc comparisons were made using the Newman-Keuls test. Skewed data, which violate the distribution requirement of the ANOVA, were subjected to arcsine, square root, or logarithmic transformations (Howell, 1987).

\section{General Results}

Tracings of the outlines of the lesions in individual animals were made based on the extent of neuronal loss in the Cresyl Violet-stained sections. Photomicrographs of representative lesions are shown in Figure 3. Figure 4 shows the extent of the lesions, which were highly similar to those obtained in previous studies using identical lesion parameters (Bussey et al., 1996; Muir, Everitt, \& Robbins, 1996). In no animal was there obvious or gross damage to the hippocampus or cingulum bundle.

As shown in Figures 3 and 4, lesions of the medial frontal cortex were well placed and extended from approximately $+4.2 \mathrm{~mm}$ from bregma to the genu of the corpus callosum, thus including areas $\mathrm{Cg} 1$ and $\mathrm{Cg} 3$ according to the nomenclature of Zilles (1985). Damage to regions posterior to the genu was rare and, when occurring, it was observed as only minimal cell loss. In the majority of cases, damage was greatest in the rostralmost areas of medial frontal cortex. Anterior cingulate cortex lesions extended from the genu of the corpus callosum to approximately $-1.0 \mathrm{~mm}$ from bregma and removed areas $\mathrm{Cg} 1$ and $\mathrm{Cg} 2$. Posterior cingulate cortex lesions extended from $-1.8 \mathrm{~mm}$ from bregma to approximately $-4.8 \mathrm{~mm}$ and revealed extensive cell loss in areas RSG and RSA according to Zilles (1985).

\section{Experiment 1a: Effects of Anterior and Posterior Cingulate Cortex Lesions on Conditional Visual Discrimination Learning}

\section{Method}

\section{Subjects}

Thirty-two rats were assigned to one of four groups-shamoperates for anterior cingulate cortex (SHAMant) $(n=5)$, shamoperates for posterior cortex (SHAMpos) $(n=5)$, anterior cingulate cortex (ANT) $(n=12)$, or posterior cingulate cortex (POS) $(n=10)$-and the appropriate surgeries performed (see the General Method section).

\section{Behavioral Procedures}

Rats were required to learn a rule of the type, "If Stimulus A, then go left, if Stimulus B, then go right." For this task, a three-window mask was used. The three windows were raised the same distance from the floor, one located centrally and the other two located to the right and left of the central window. A trial began with the presentation of one of two discriminative stimuli, determined pseudorandomly, in the central window. The rat was required to make a nose poke to this stimulus. Immediately after the disappearance of the discriminative stimulus, two white squares (the choice stimuli) appeared in the left and right windows. A nose poke to the appropriate square (left or right depending on the discriminative stimulus) was rewarded as described earlier; an incorrect response was punished with a time-out. Both discriminative stimuli were presented an equal number of times during a session; therefore, left and right responses were correct an equal number of times. If the rat did not respond to the choice stimuli within a 2-s limited hold period, the choice stimuli disappeared and the houselights were extinguished for a 5-s time-out period. This ensured that a rat's response to a choice stimulus was always a head turn to the right or left while rearing, following finally by a nose poke to the stimulus. Each session consisted of 100 trials.

\section{Results}

\section{Histology}

Histological analysis of the lesions revealed incomplete lesions in 2 animals in the ANT group and 1 animal in the POS lesion group. For the ANT group, both animals had lesions that were unilateral; in the POS animals, infusion of the excitotoxin did not result in neuronal loss. The data from these animals were therefore excluded from subsequent behavioral analyses.

\section{Behavioral Results}

Two animals, one in the POS group and one in the SHAMant group, became ill during behavioral testing. The final group membership was as follows: SHAMant $=4$, SHAMpos $=5$, ANT $=10$, and $\mathrm{POS}=8$.

Errors to criterion. Bussey et al. (1996) reported on the differential effects of cingulate cortex lesions on a conditional visual discrimination carried out in an operant chamber, depending on whether acquisition was examined in the early or late stages of learning (see the introduction). Accordingly, acquisition data from this experiment were analyzed in terms of the number of errors committed by the animals during three learning stages: (a) chance performance to $61 \%$ correct responses, (b) 61-70\% correct responses, and (c) 70-85\% correct choices (all criteria require performance at the stated level for two sessions consecutively). These were the same percentage criteria used by Bussey et al. (1996) and Muir, Bussey, Everitt, and Robbins (1996), except that whereas a criterion of $59 \%$ correct choice out of 128 trials was used to delineate the first stage in these studies, $61 \%$ was used in the current analysis because this is the level of performance that is significantly above chance for a session comprising 100 , rather than 128 , trials.

Preliminary analysis of the SHAMant and SHAMpos groups revealed no significant effect of lesion, $F(1,7)=$ 

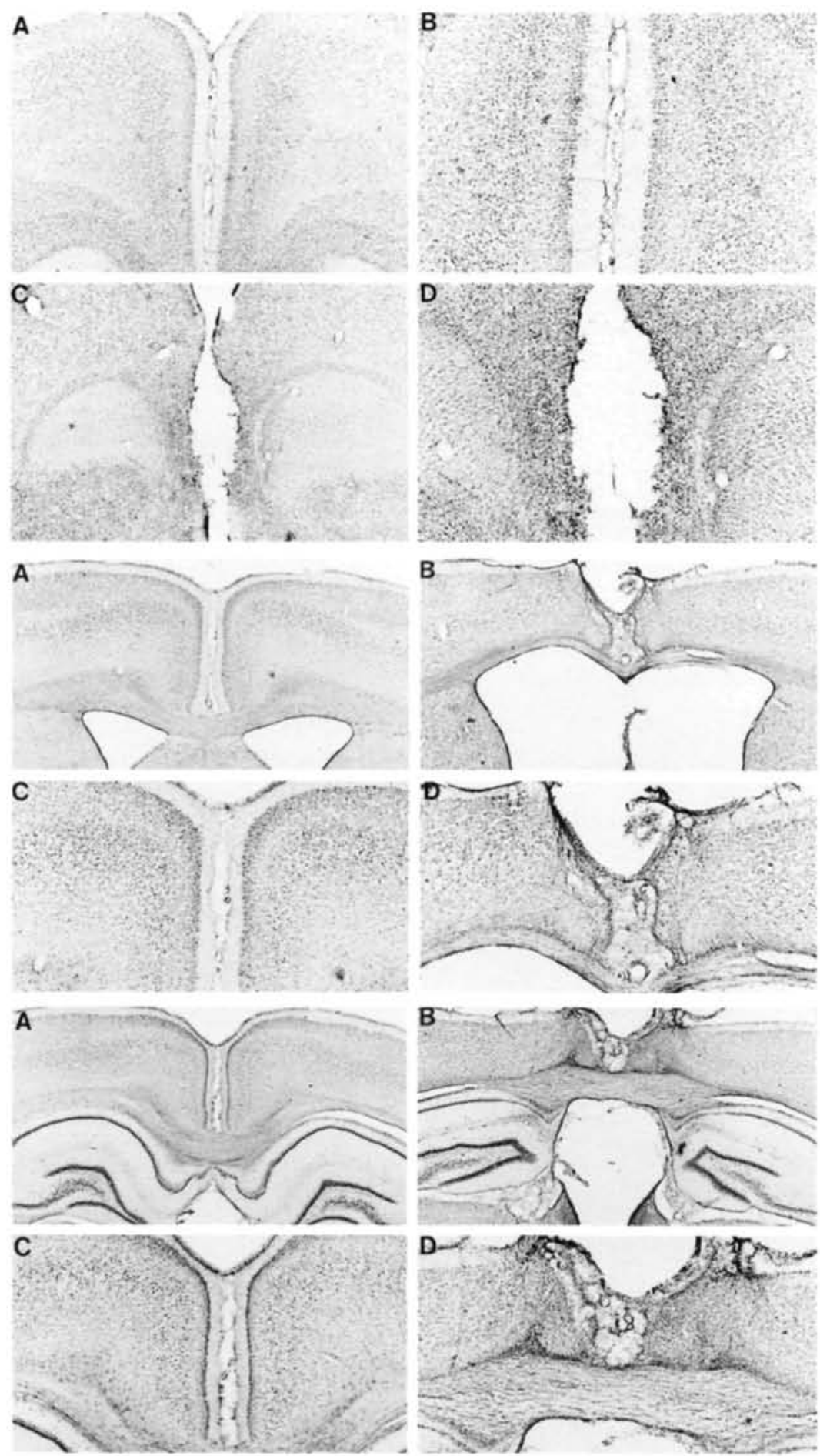

Figure 3. Photomicrographs showing the extent of the lesions of the medial frontal (top), anterior cingulate (middle), and posterior cingulate (bottom) cortices. Top: The material shown in A and B is from sham-operated animals at, respectively, low and high magnification. C (low magnification) and D (high magnification) show medial frontal lesions in which neuronal damage is observed only in regions rostral to the genu of the corpus callosum. Middle: The material shown in $\mathrm{A}$ and $\mathrm{C}$ is from sham-operated animals, respectively, at low and high magnification. B (low magnification) and D (high magnification) show lesions of the anterior cingulate cortex in which marked neuronal loss extends caudally from the genu of the corpus callosum, in most cases producing only minimal loss pre-genu. Some ventricular enlargement was observed in these animals. Bottom: The material shown in B (low magnification) and D (high magnification) is typical of lesions observed in the posterior cingulate cortex-lesioned animals in which neuronal loss is confined to retrosplenial agranular cortex and retrosplenial granular cortex areas according to Zilles (1985). A and C show sham-operated rats at low and high magnification, respectively. 

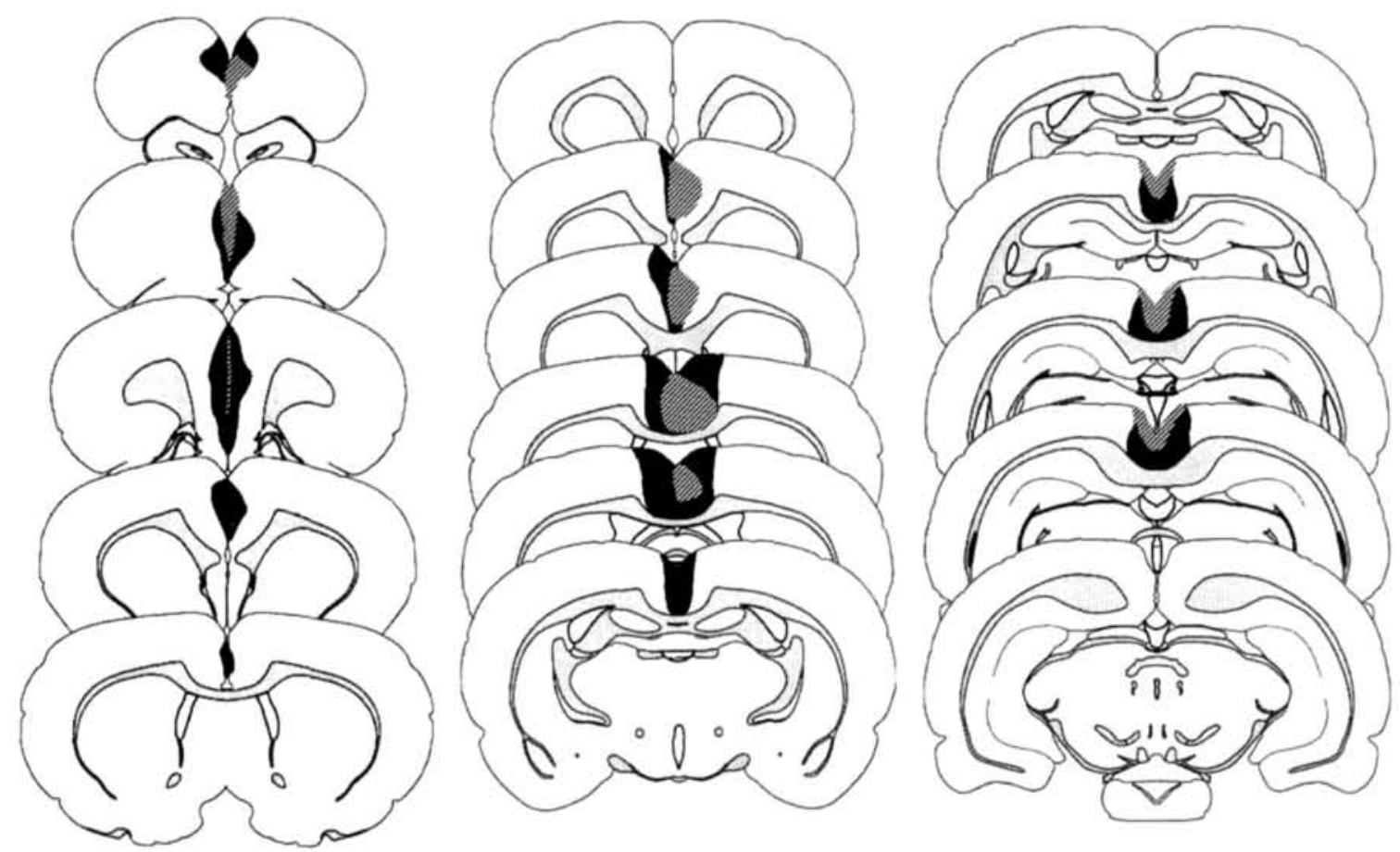

Figure 4. Reconstructions of the medial frontal (left), anterior cingulate (middle), and posterior cingulate (right) cortex lesions at various anteroposterior levels (medial frontal cortex $=+4.2$ to $+1.2 \mathrm{~mm}$ from bregma; anterior cingulate cortex $=+2.2$ to $-1.8 \mathrm{~mm}$ from bregma; and posterior cingulate cortex $=-1.8$ to $-5.6 \mathrm{~mm}$ from bregma). Black shading indicates the extent of the largest lesion within each group; cross-hatched shading indicates the smallest lesion. Drawings of sections are from Swanson (1992).

$0.352, p>.05$, a significant effect of learning stage, $F(2,14)=24.8, p<.001$, and no significant Lesion $\times$ Learning Stage interaction, $F(2,14)=0.106, p>.05$. Because there were no significant differences between these groups, we treated these animals as a single SHAM group during subsequent analyses.

The results for the acquisition of this task for sham and lesion groups are shown in Figure 5. A two-way ANOVA revealed no significant effect of lesion, $F(2,24)=1.94, p>$ .05 , a significant effect of learning stage, $F(2,48)=113.8$, $p<.001$, and a significant Lesion $\times$ Learning Stage interaction, $F(4,48)=2.94, p<.05$. Newman-Keuls post hoc analysis of the significant interaction revealed that animals in the POS group made significantly more errors in the final stage of learning the task (i.e., progressing from $70 \%$ to $85 \%$ correct) than did animals in either the SHAM or the ANT groups (both comparisons, $p<.01$ ). Animals in the ANT group did not differ significantly from animals in the SHAM control group at this stage, and there were no differences between groups at either of the other two stages of learning.

Percentage of bias. There were no significant differences between groups in terms of the percentage of bias to respond to one particular response side, $F(2,24)=1.87$, $p>.05(\mathrm{SHAM}=10.9 \%, \mathrm{ANT}=7.2 \%$, and $\mathrm{POS}=6.1 \%)$.

Choice latency. Choice latency for the conditional task was calculated as the time taken from sample poke to choice

\section{Visuospatial Conditional Task}

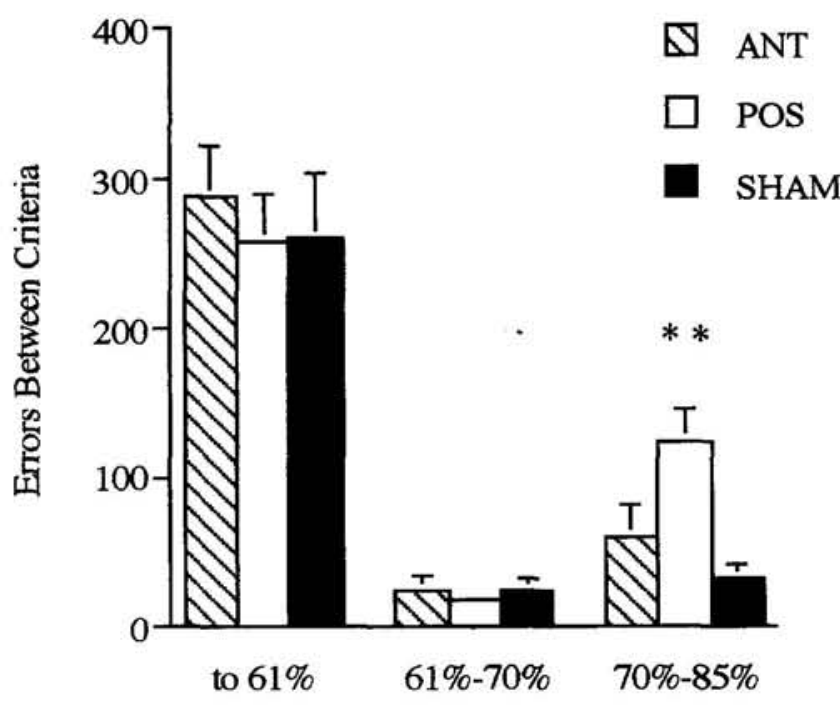

Figure 5. Errors required by anterior cingulate-lesioned (ANT), posterior cingulate-lesioned (POS), and sham-operated control (SHAM) animals to progress between criteria associated with various stages of learning during the acquisition of the conditional visual discrimination task (Experiment 1a). $* *$ Significant difference from SHAM animals at the .01 level. Error bars show \pm 1 SEM. 
poke. Because the sample stimulus offset time was $0.5 \mathrm{~s}$ after the sample poke, and rats typically did not move to make a choice until sample offset, the actual choice latency was approximately $0.5 \mathrm{~s}$ less than that reported. An ANOVA revealed that there were no differences between groups in terms of choice latency, $F(2,24)=0.10, p>.05$ $(\mathrm{SHAM}=1.07 \mathrm{~s}, \mathrm{ANT}=1.06 \mathrm{~s}$, and $\mathrm{POS}=1.10 \mathrm{~s})$.

Magazine latency. An ANOVA showed a significant main effect of lesion on magazine latency, $F(2,24)=3.91$, $p<.05$. Newman-Keuls post hoc analysis revealed that the average magazine latency for the ANT group was significantly greater than that for either the SHAM or the POS groups $(p<.05)($ SHAM $=1.33 \mathrm{~s}$, ANT $=1.61 \mathrm{~s}$, and $\mathrm{POS}=1.27 \mathrm{~s})$.

\section{Experiment 1b: Effects of Medial Frontal \\ (MF) Cortex Lesions on Conditional Visual Discrimination Learning}

\section{Method}

\section{Subjects}

Twelve rats were assigned to one of two groups-SHAM $(n=6)$ or MF $(n=6)$-and the appropriate surgeries performed (see the General Method section).

\section{Behavioral Procedures}

The behavioral procedures were the same as in Experiment 1a.

\section{Results}

\section{Errors to Criterion}

Comparison of the SHAM groups in this experiment and Experiment 1a revealed a distinct difference between the performance of these two groups, probably because of noticeable differences in temperature and humidity in the testing rooms at the times these two experiments were run (the total number of errors is discussed later). The data from these two cohorts are thus presented separately. Data from this experiment were analyzed as in Experiment 1a. A two-way ANOVA revealed no significant effect of lesion on this response measure, $F(1,10)=0.91, p>.05$, a significant effect of learning stage, $F(2,20)=25.17, p<$ .001 , and no significant Lesion $\times$ Learning Stage interaction, $F(2,20)=1.75, p>.05$. Thus, there was no significant difference between animals in the MF group and animals in the SHAM group at any stage of acquisition of this task; if anything, animals of the MF group made fewer errors than did animals of the SHAM group (the total errors for the SHAM and MF groups were 520.2 and 392.8, respectively).

\section{Percentage of Bias}

An ANOVA revealed that there were no significant differences between groups in terms of percentage of bias, $F(1,10)=0.175, p>.05(\mathrm{SHAM}=11.6 \%$ and $\mathrm{MF}=$ $10.6 \%)$.

\section{Choice Latency}

Animals in the MF group responded significantly faster to the choice stimuli than did animals in the SHAM group, $F(1,10)=5.24, p<.05($ SHAM $=1.06 \mathrm{~s}$ and $\mathrm{MF}=0.94 \mathrm{~s})$.

\section{Magazine Latency}

There also were no differences between groups in terms of magazine latency, $F(1,10)=0.121, p>.05($ SHAM $=1.04 \mathrm{~s}$ and $\mathrm{MF}=1.33 \mathrm{~s}$ ).

Experiment 2a: Effects of Anterior and Posterior Cingulate Cortex Lesions on Eight-Pair Concurrent Discrimination Learning

\section{Method}

\section{Subjects}

The subjects were the same as those used in Experiment 1a.

\section{Behavioral Procedures}

Rats were required to learn the correct, reinforced stimulus in each of eight pairs. Each of the eight stimulus pairs was presented 15 times during a session, resulting in a total of 120 trials per session. The sequence of presentation of stimulus pairs for each session was determined in a pseudorandom fashion. Each stimulus appeared in the left and right windows an approximately equal number of times ( 7 lefts and 8 rights for one stimulus of each pair and 8 lefts and 7 rights for the other). The correct stimulus in each pair was identical for each rat.

\section{Results}

\section{Errors to Criterion}

Preliminary analysis of the SHAMant and SHAMpos groups' data revealed no significant effect of lesion, $F(1,7)=$ $3.43, p>.05$. Because there were no significant differences between these groups, we treated these animals as a single SHAM group during subsequent analyses.

The number of errors required to attain criterion performance of $85 \%$ correct choices on two consecutive sessions for the lesion and sham groups are shown in Figure 6. A one-way ANOVA revealed a significant effect of lesion, $F(2,24)=8.57, p<.01$. Newman-Keuls post hoc analysis showed significant differences between the ANT and SHAM groups $(p<.05)$, between the POS and SHAM groups $(p<$ $.05)$, and between the ANT and POS groups $(p<.01)$. Thus, although animals in the ANT group committed significantly more errors during acquisition than did animals in the SHAM group, animals of the POS group made significantly fewer errors than did animals in the SHAM group.

\section{Percentage of Bias}

An ANOVA revealed that there were no significant differences between groups in terms of percentage of bias, 


\section{8-Pair Concurrent Discrimination}

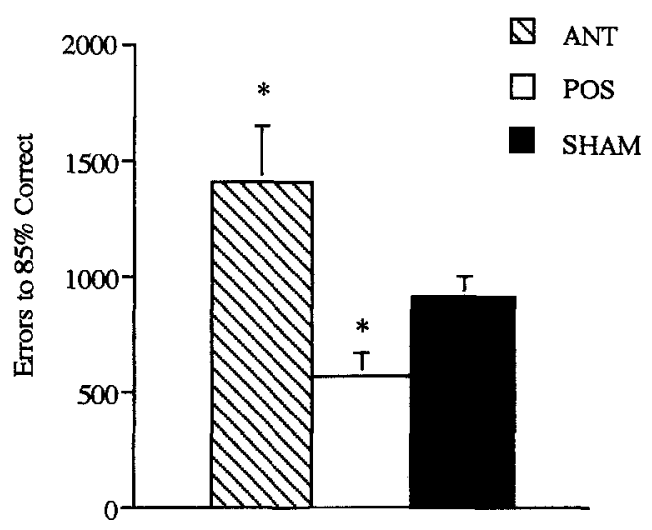

Figure 6. Errors required by anterior cingulate-lesioned (ANT) and posterior cingulate-lesioned (POS) animals and sham-operated control (SHAM) animals to attain criterion performance of $85 \%$ correct on the eight-pair concurrent discrimination task (Experiment 2a). *Significant difference from SHAM animals at the .05 level.

$F(2,24)=0.233, p>.05(\mathrm{SHAM}=10.1 \%, \mathrm{ANT}=10.7 \%$, and $\mathrm{POS}=9.8 \%$ ).

\section{Choice Latency}

There were no differences between groups in terms of choice latency, $F(2,24)=1.17, p>.05(\mathrm{SHAM}=4.4 \mathrm{~s}$, $\mathrm{ANT}=3.23 \mathrm{~s}$, and POS $=3.87 \mathrm{~s}$ ).

\section{Magazine Latency}

There also were no significant differences between groups in terms of magazine latency, $F(2,24)=1.52, p>.05$ $($ SHAM $=1.67 \mathrm{~s}, \mathrm{ANT}=1.66 \mathrm{~s}$, and POS $=1.81 \mathrm{~s})$.

\section{Experiment 2b: Effects of Medial Frontal Cortex Lesions on Eight-Pair Concurrent Discrimination Learning}

\section{Method}

\section{Subjects}

The subjects were the same as those used in Experiment $1 \mathrm{~b}$.

\section{Behavioral Procedures}

The behavioral procedures were the same as those used in Experiment 2a.

\section{Results}

\section{Errors to Criterion}

A one-way ANOVA revealed that there was no significant effect of lesion in terms of errors to criterion, $F(1,10)=$ $0.31, p>.05($ SHAM $=1,533$ and $\mathrm{MF}=1,274)$.

\section{Percentage of Bias}

There were no significant differences between groups in terms of percentage of response bias, $F(1,10)=0.030, p>$ $.05($ SHAM $=14.2 \%$ and $M F=13.8 \%)$.

\section{Choice Latency}

An ANOVA revealed that there were no significant differences between groups in terms of choice latency, $F(1$, $10)=4.79, p>.05($ SHAM $=5.98 \mathrm{~s}$ and $\mathrm{MF}=5.98 \mathrm{~s})$.

\section{Magazine Latency}

There were no significant differences between groups in terms of magazine latency, $F(1,10)=1.78, p>.05$ $(\mathrm{SHAM}=2.08 \mathrm{~s}$ and $\mathrm{MF}=1.71 \mathrm{~s})$.

Experiment 3: Effects of Anterior Cingulate, Posterior Cingulate, and Medial Frontal Cortex Lesions on "Difficult" One-Pair Discrimination and Reversal Learning

\section{Method}

\section{Subjects}

Forty-two rats were assigned to one of six groupsSHAMant $(n=4)$, SHAMpos $(n=4)$, SHAMmf $(n=4)$, ANT $(n=10)$, POS $(n=10)$, or MF $(n=10)$-and the appropriate surgeries performed (see the General Method section). During training, 2 animals from the ANT group, 1 from the MF group, 1 from the SHAMant group, and 1 from the SHAMmf group became ill. In addition, 1 animal from the ANT group died after surgery. Therefore, final group numbers were as follows: SHAMant $=3$, SHAMpos $=4$, SHAMmf $=3$, ANT $=7, \operatorname{POS}=10$, and $\mathrm{MF}=9$.

\section{Behavioral Procedures}

In the one-pair discrimination task, the same pair of stimuli were presented on every trial. One stimulus was designated the $S+$ and the other the $\mathrm{S}-$. Which stimulus was on the left and which was on the right was determined pseudorandomly. The stimuli in this experiment were designed to be difficult to discriminate; although they were different in color (green vs. blue), they were approximately the same size and luminance. A nose poke to the $S+$ was rewarded with tone, magazine light, and sucrose pellet. A nose poke to the $\mathrm{S}$ - was followed by extinction of the houselight for a 5 -s time-out period.

Once a rat had attained a criterion of $85 \%$ correct responses for two sessions consecutively, it was moved on to the first reversal, in which the stimulus formerly designated as the $\mathrm{S}+$ became the $\mathrm{S}-$ and vice versa. Once a rat had attained the $85 \%$ criterion on a reversal, the $\mathrm{S}+$ and $\mathrm{S}-$ were again reversed. A total of three reversals were given.

\section{Results}

\section{Errors to Criterion}

Preliminary analysis of the SHAMant, SHAMpos, and SHAMmf groups revealed no significant effect of lesion, 
$F(2,7)=3.13, p>.05$, a significant effect of task (acquisition plus three reversals), $F(3,21)=21.7, p<.001$, and no significant Lesion $\times$ Task interaction, $F(3,21)=$ $1.47, p>.05$. Because there were no significant differences between these groups, we treated these animals as a single SHAM group during subsequent analyses.

Figure 7 shows errors committed by animals during acquisition of the discrimination and the three reversals of that discrimination for the lesion and sham groups. A two-way ANOVA revealed a significant main effect of lesion, $F(3 ; 32)=3.09, p<.05$, a significant main effect of task, $F(3,96)=73.8, p<.001$, and a significant Lesion $\times$ Task interaction, $F(9,96)=2.11, p<.05$. Newman-Keuls post hoc analysis revealed that the main effect of lesion resulted from POS animals committing fewer errors across acquisition and reversals than MF animals. Newman-Keuls post hoc analysis of the Lesion $\times$ Task interaction revealed that MF animals committed significantly more errors during each of the three reversals than either of the SHAM (Reversal 1, $p<.05$; Reversal 2, $p<.01$; and Reversal 3, $p<.01$ ), ANT (Reversal 1, $p<.05$; Reversal 2, $p<.05$ and Reversal 3, $p<.05$ ), or POS (Reversal 1, $p<.05$; Reversal 2, $p<.01$, and Reversal 3, $p<.01$ ) groups.

Data were further analyzed according to the method of Dias et al. (1996), based on a method used by Jones and Mishkin (1972). In this analysis, errors during reversal learning were broken down into two learning stages: errors committed before the attainment of chance-level performance (39\% correct for 100 trials) and errors committed between $39 \%$ and $85 \%$ correct responses. Jones and Mishkin regarded errors made during the first stage of learning as being indicative of perseverative responses to the previously reinforced stimulus.

The number of errors committed during these two learning stages (errors to $39 \%$; errors between $39 \%$ and $85 \%$ ) are shown in Figure 8. (All criteria require performance at the stated level for two sessions consecutively.) An ANOVA revealed that there was no significant effect of lesion, $F(3$, $32)=2.55, p>.05$, but that there was a significant effect of learning stage, $F(1,32)=79.59, p<.001$, and a significant

\section{1-Pair Discrimination and Reversal: "Difficult"}

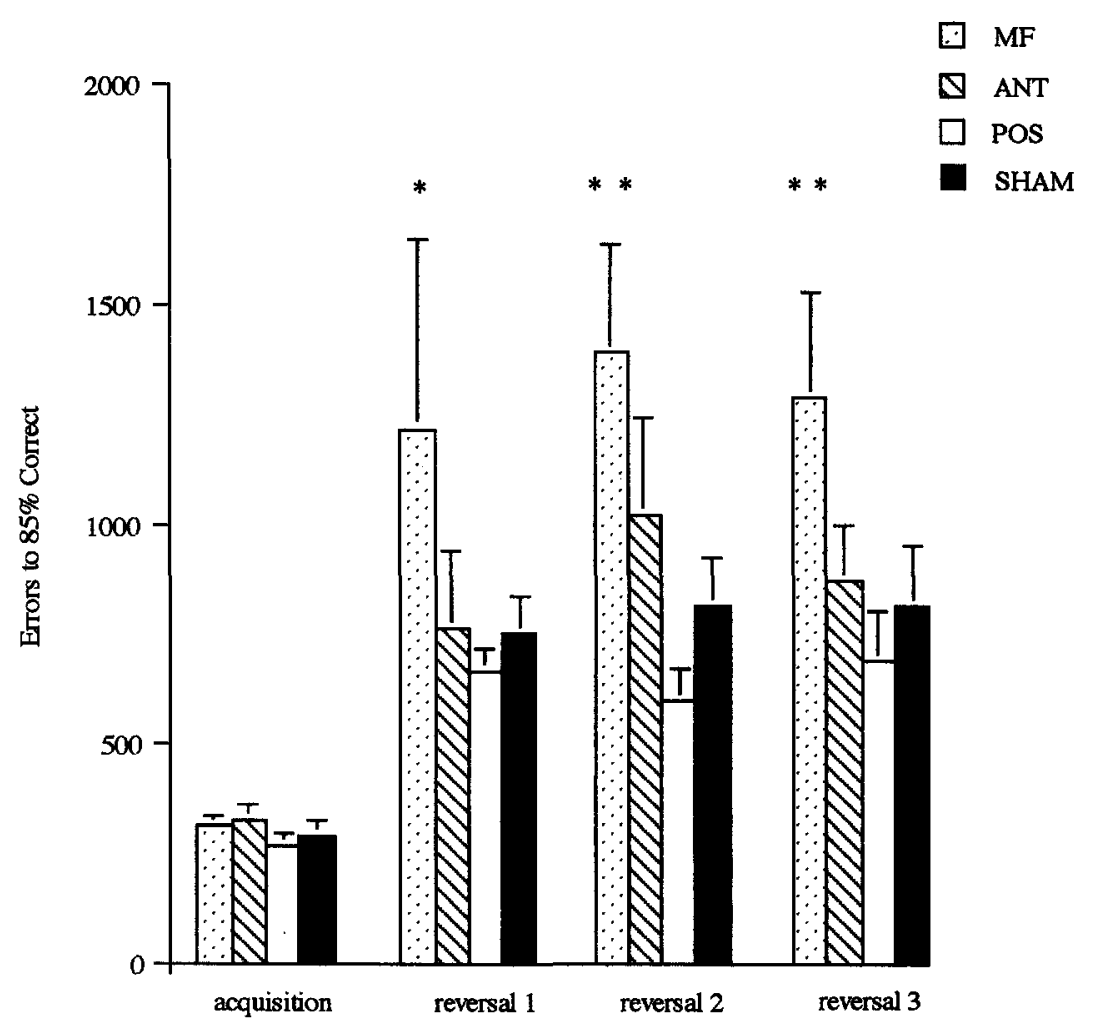

Figure 7. Errors required by medial frontal-lesioned (MF), anterior cingulate-lesioned (ANT), and posterior cingulate-lesioned (POS) animals and sham-operated control (SHAM) animals, to attain criterion performance of $85 \%$ correct on the acquisition and three reversals of a one-pair shape discrimination using stimuli that were difficult to discriminate (Experiment 3 ). *Significant difference from SHAM animals at the .05 level. ${ }^{*}$ Significant difference from SHAM animals at the .01 level. 


\section{1-Pair Discrimination Reversal: "Difficult" \\ Stages of Acquisition}

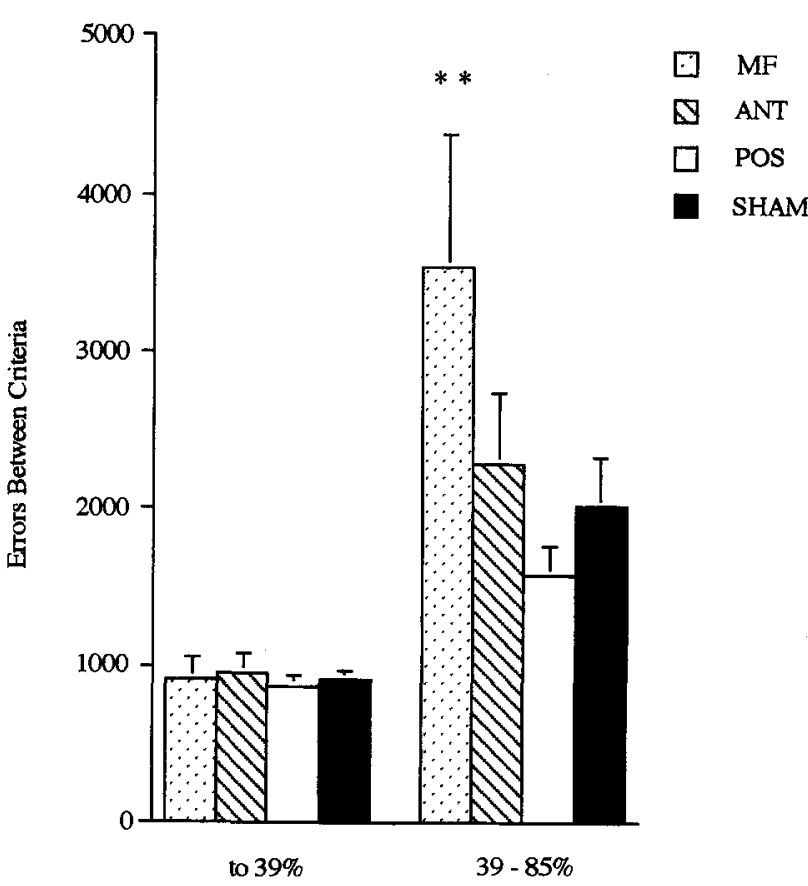

Figure 8 . Errors required by medial frontal-lesioned (MF), anterior cingulate-lesioned (ANT), and posterior cingulate-lesioned (POS) animals and sham-operated control (SHAM) animals to progress between criteria associated with two stages of learning summed across three reversals of a one-pair shape discrimination using stimuli that were difficult to discriminate (Experiment 3 ). **Significant difference from SHAM animals at the .01 level.

Lesion $\times$ Learning Stage interaction, $F(3,32)=3.95, p<$ .05 . Newman-Keuls post hoc analysis of the interaction revealed that during the second stage of learning (39-61\%), the MF animals committed significantly more errors than did the SHAM, POS, or ANT animals $(p<.01)$, which did not differ from each other. There were no differences between any of the groups in the number of errors committed during the initial stage of learning (errors to $39 \%$ ).

Thus, animals with medial frontal cortex lesions were significantly impaired in reversal learning on all three reversals. Further analysis suggested that this impairment did not result from these animals making perseverative responses to the previously reinforced stimulus.

\section{Percentage of Bias}

There was no main effect of lesion, $F(3,32)=0.80, p>$ .05 , or Lesion $\times$ Task interaction, $F(9,96)=1.28, p>.05$. However, there was a significant main effect of task, $F(3$, 96) $=7.22, p<.001$, indicating that percentage of bias decreased as training proceeded across reversals (mean values: acquisition, SHAM $=14.2 \%$, ANT $=12.7 \%$, POS $=13.3 \%$, and MF $=12.5 \%$; Reversal 1, SHAM $=$ $13.9 \%$, ANT $=11.9 \%$, POS $=15.7 \%$, and $\mathrm{MF}=11.6 \%$; Reversal 2, SHAM $=10.6 \%$, ANT $=12.8 \%$, POS $=10.2 \%$, and $\mathrm{MF}=10.5 \%$; and Reversal 3, SHAM $=9.4 \%$, ANT $=$ $10.0 \%, \operatorname{POS}=12.5 \%$, and $\mathrm{MF}=9.8 \%$ ).

\section{Choice Latency}

There was no significant main effect of lesion, $F(3,32)=$ $2.66, p>.05$, or Lesion $\times$ Task interaction, $F(9,96)=1.12$, $p>.05$. However, there was a significant main effect of task, $F(3,96)=16.0, p<.001$, indicating that choice latency decreased as training proceeded across reversals (mean values: acquisition, SHAM $=7.29 \mathrm{~s}$, ANT $=6.03 \mathrm{~s}$, POS $=10.4 \mathrm{~s}$, and MF $=10.3 \mathrm{~s} ;$ Reversal 1, SHAM $=7.21$ $\mathrm{s}, \mathrm{ANT}=4.01 \mathrm{~s}, \mathrm{POS}=5.60 \mathrm{~s}$, and MF $=5.11 \mathrm{~s}$; Reversal 2 , SHAM $=4.95 \mathrm{~s}$, ANT $=3.19 \mathrm{~s}$, POS $=4.27 \mathrm{~s}$, and $\mathrm{MF}=4.44 \mathrm{~s}$; and Reversal 3, SHAM $=4.36 \mathrm{~s}, \mathrm{ANT}=3.02$ $\mathrm{s}, \mathrm{POS}=4.72 \mathrm{~s}$, and MF $=4.32 \mathrm{~s}$ ).

\section{Magazine Latency}

There was no significant main effect of lesion, $F(3,32)=$ $0.490, p>.05$, or of task, $F(3,96)=0.238, p>.05$, nor was there a significant Lesion $\times$ Task interaction, $F(9,96)=$ $1.43, p>.05$ (mean values: acquisition, $\mathrm{SHAM}=1.48 \mathrm{~s}$, ANT $=1.60 \mathrm{~s}$, POS $=1.50 \mathrm{~s}$, and MF $=1.82 \mathrm{~s} ;$ Reversal 1 , SHAM $=1.83 \mathrm{~s}$, ANT $=1.46 \mathrm{~s}$, POS $=1.29 \mathrm{~s}$, and MF $=$ $1.61 \mathrm{~s}$; Reversal 2, SHAM $=1.60 \mathrm{~s}$, ANT $=1.33 \mathrm{~s}$, POS $=$ $1.71 \mathrm{~s}$, and MF $=1.39 \mathrm{~s}$; and Reversal 3, SHAM $=1.78 \mathrm{~s}$, $\mathrm{ANT}=1.43 \mathrm{~s}, \mathrm{POS}=1.51 \mathrm{~s}$, and $\mathrm{MF}=1.48 \mathrm{~s}$ ).

\section{Experiment 4a: Effects of Anterior and Posterior} Cingulate Cortex Lesions on One-Pair Discrimination and Reversal Learning Using "Easy" Stimuli

In Experiment 3 we found that animals with lesions of the medial frontal cortex were impaired in reversal learning. However, further analysis did not provide evidence for perseveration in these animals. Instead, the pattern of results may suggest a deficit in stimulus-reward learning (see Jones \& Mishkin, 1972). To investigate further the nature of the reversal learning deficit exhibited by medial frontal cortexlesioned animals, we examined one-pair discrimination and reversal learning with easily discriminable stimuli. This manipulation should reduce the perceptual demands of the task and thus increase the premium on stimulus-reward learning. In this experiment, the effects of lesions of the anterior or posterior cingulate cortex were examined; in Experiment $4 \mathrm{~b}$. we tested medial frontal cortex-lesioned animals on this task.

\section{Method}

\section{Subjects}

Twenty-four rats were assigned to one of three groupsSHAMant $(n=4)$, SHAMpos $(n=4)$, ANT $(n=8)$, and POS $(n=8)$ and the appropriate surgeries performed (see the General 
Method section). One animal in the POS group died after surgery. Therefore, the final group numbers were as follows: SHAMant $=$ 4, SHAMpos $=4$, ANT $=8$, and POS $=7$.

\section{Behavioral Procedures}

Behavioral testing proceeded as in Experiment 3, except that stimuli were chosen to maximize discriminability. Thus, the S+ and $\mathrm{S}-$ differed in color and size, one had jagged edges and one had smooth edges, and one featured horizontal lines and the other had more vertical lines. After acquisition of this discrimination, a single reversal of the discrimination was performed.

\section{Results}

\section{Errors to Criterion}

Preliminary analysis of the SHAMant and SHAMpos groups' data revealed no significant effect of lesion, $F(1,6)=$ $2.71, p>.05$, a significant effect of task, $F(1,6)=20.6, p<$ .01 , and no significant Lesion $\times$ Task interaction, $F(1,6)=$ $0.675, p>.05$. Because there were no significant different differences between these groups, we treated these animals as a single SHAM group during subsequent analyses.

An ANOVA revealed that there was no significant main effect of lesion, $F(2,20)=0.955, p>.05$, a significant main effect of task, $F(1,20)=54.37, p<.001$, and no significant Lesion $\times$ Task interaction, $F(2,20)=0.587, p>.05$ (mean values: acquisition, $\mathrm{SHAM}=139.2, \mathrm{ANT}=158.2$, and POS $=144.0 ;$ reversal, $\mathrm{SHAM}=313.3, \mathrm{ANT}=364.8$, and POS $=416.1$ ).

\section{Percentage of Bias}

There was no main effect of lesion, $F(2,20)=0.316, p>$ .05 , or task, $F(1,20)=0.808, p>.05$, nor was there a significant Lesion $\times$ Task interaction, $F(2,20)=0.211, p>$ .05 (mean values: acquisition, $\mathrm{SHAM}=13.9 \%$, ANT $=$ $13.3 \%$, and POS $=13.9 \%$; reversal, SHAM $=16.8 \%$, $\mathrm{ANT}=16.1 \%$, and $\mathrm{POS}=13.9 \%$ ).

\section{Choice Latency}

An ANOVA revealed that there was no significant main effect of lesion on choice latency, $F(2,20)=1.93, p>.05$, although there was a main effect of task, $F(1,20)=36.0$, $p<.001$, indicating that animals in all groups were slower to approach the choice stimuli during reversal learning. There also was a significant Lesion $\times$ Task interaction, $F(2$, $20)=10.51, p<.001$. Newman-Keuls post hoc analysis revealed that this was caused by the POS animals having significantly longer choice latencies during reversal learning than the SHAM or ANT animals $(p<.05)$ (mean values: acquisition, $\mathrm{SHAM}=5.58 \mathrm{~s}$, ANT $=5.99 \mathrm{~s}$, and POS $=$ $4.23 \mathrm{~s}$; reversal, SHAM $=8.61 \mathrm{~s}$, ANT $=7.14 \mathrm{~s}$, and POS $=$ $13.3 \mathrm{~s})$.

\section{Magazine Latency}

There was a significant main effect of lesion on magazine latency, $F(2,20)=6.75, p<.01$, a significant main effect of task, $F(1,20)=7.96, p<.05$, and a significant Lesion $\times$ Task interaction, $F(2,20)=5.22, p<.05$. Newman-Keuls post hoc analysis of the interaction revealed that the average magazine latency for the ANT group was greater than that for the SHAM group $(p<.05)$; that the average magazine latency for the SHAM group was greater than that for the POS group $(p<.05)$; and that the average magazine latency for the ANT group was greater than that for the POS group $(p<.01)$ (mean values: acquisition, SHAM $=1.45 \mathrm{~s}$, ANT $=1.66 \mathrm{~s}$, and POS $=1.09 ;$ reversal, SHAM $=1.24 \mathrm{~s}$, ANT $=1.27 \mathrm{~s}$, and POS $=1.18 \mathrm{~s}$ ).

Experiment 4b: Effects of Medial Frontal Cortex Lesions on One-Pair Discrimination and Reversal Learning Using Easy Stimuli

\section{Method}

\section{Subjects}

Eighteen rats were assigned to one of two groups-MF $(n=10)$ or SHAM $(n=8)$-and the appropriate surgeries performed (see the General Method section). One animal in the MF group died after surgery. Therefore, the final group numbers were as follows: $\mathrm{MF}=9$ and $\mathrm{SHAM}=8$.

\section{Behavioral Procedures}

Behavioral testing proceeded as in Experiment 4a. However, because medial frontal-lesioned animals were found to be unimpaired after a single reversal, and because in Experiment 3 the mildest impairment was observed on the first reversal, a second reversal was added to test whether a deficit would emerge.

\section{Results}

\section{Errors to Criterion}

An ANOVA revealed that there was no significant main effect of lesion, $F(1,15)=0.1142, p>.05$, a significant main effect of task, $F(2,30)=50.09, p<.001$, and no significant Lesion $\times$ Task interaction, $F(2,30)=0.692, p>$ .05 (mean values: acquisition, SHAM $=95.3$ and MF $=$ 94.3; Reversal 1, SHAM $=267.3$, and $\mathrm{MF}=225.0$; and Reversal 2, SHAM $=224.3$ and $M F=184.9$ ).

\section{Percentage of Bias}

There was no significant main effect of lesion, $F(1,15)=$ $0.449, p>.05$, or Lesion $\times$ Task interaction, $F(2,30)=$ $1.82, p>.05$. However, there was a significant main effect of task, $F(2,30)=6.14, p<.01$, indicating that percentage of bias increased as training proceeded across reversals (mean values: acquisition, SHAM $=8.25 \%$ and $\mathrm{MF}=$ $8.25 \%$; Reversal 1, SHAM $=12.3 \%$ and $\mathrm{MF}=13.1 \%$; and Reversal 2, SHAM $=15.6 \%$ and $\mathrm{MF}=10.6 \%$ ).

\section{Choice Latency}

There was no main effect of lesion, $F(1,15)=5.33, p>$ .05 , or task, $F(2,30)=2.72, p>.05$, nor was there a 
significant Lesion $\times$ Task interaction, $F(2,30)=0.514, p>$ .05 (mean values: acquisition, SHAM $=5.50 \mathrm{~s}$ and $\mathrm{MF}=7.79 \mathrm{~s} ;$ Reversal 1, SHAM $=5.27 \mathrm{~s}$ and $\mathrm{MF}=5.93$ $\mathrm{s}$; and Reversal 2, SHAM $=3.84 \mathrm{~s}$ and $\mathrm{MF}=5.63 \mathrm{~s}$ ).

\section{Magazine Latency}

There was no significant main effect of lesion, $F(1,15)=$ $0.107, p>.05$, or task, $F(2,30)=2.87, p>.05$, nor was there a significant Lesion $\times$ Task interaction, $F(2,30)=$ $0.378, p>.05$ (mean values: acquisition, $\mathrm{SHAM}=1.76 \mathrm{~s}$ and $\mathrm{MF}=1.85 \mathrm{~s}$; Reversal 1, SHAM $=1.72 \mathrm{~s}$ and $\mathrm{MF}=$ $1.46 \mathrm{~s}$; and Reversal $2, \mathrm{SHAM}=1.36 \mathrm{~s}$ and $\mathrm{MF}=1.29 \mathrm{~s}$ ).

\section{Discussion}

Our experiments, the results of which are summarized in Table 1, demonstrate a triple functional dissociation between the anterior cingulate, posterior cingulate, and medial frontal cortices of the rat. The excitotoxic lesioning technique was effective in producing discrete lesions of these regions, with no obvious or gross damage to the hippocampus or cingulum bundle. Anterior cingulate cortex lesions led to an impairment in the acquisition of an eight-pair concurrent discrimination task (Experiment 2a). Posterior cingulate cortex lesions produced a selective impairment in the acquisition of the visuospatial conditional discrimination task late in learning (Experiment 1a). These lesions also led to a significant facilitation of learning in the eight-pair concurrent discrimination task (Experiment 2a). Finally, animals with lesions of the medial frontal cortex were impaired in reversal learning but only late in learning and only when the stimuli used were difficult to discriminate (Experiments 3 and $4 \mathrm{~b}$ ). Because all tasks used in this research were carried out in the same apparatus, required similar responses, and used similar stimuli and the same systems of reinforcement, these effects are unlikely to have been caused by differences in perceptual, motivational, or motor functions. The results provide strong evidence that these three adjacent cortical regions, which are often treated as functionally equivalent in lesion studies, are in fact functionally distinct.

Our research also introduces the touchscreen testing

Table 1

Effects of Cortical Lesions on Visual Discrimination Tasks

\begin{tabular}{lccc}
\hline & \multicolumn{3}{c}{ Lesion type } \\
\cline { 2 - 4 } Discrimination task & MF & ANT & POS \\
\hline Conditional & NSE & NSE & Impaired \\
Eight-pair concurrent & NSE & Impaired & Facilitated \\
One pair & & & \\
"Difficult" & & & \\
Acquisition & NSE & NSE & NSE \\
Reversal & Impaired & NSE & NSE \\
"Easy" & & & \\
Acquisition & NSE & NSE & NSE \\
Reversal & NSE & NSE & NSE \\
\hline
\end{tabular}

Note. $\quad \mathrm{MF}=$ medial frontal cortex; $\mathrm{ANT}=$ anterior cingulate cortex; $\mathrm{POS}=$ posterior cingulate cortex; NSE = no significant effect. method for the rat (Bussey et al., 1994) as a valuable tool for investigating the neurobiology of learning, memory, and attention. Although the advantages of this method have been discussed in some detail elsewhere (Bussey et al., 1994), the research described here demonstrates the method's utility in lesion studies in which task parameters can be held constant across a wide variety of tasks that differ only in their cognitive demands, thus facilitating the comparative interpretation of lesion results. The effects of lesions to the anterior cingulate, posterior cingulate, and medial frontal cortices are considered separately.

\section{Effects of Anterior Cingulate Cortex Lesions}

Lesions of the anterior cingulate cortex produced a selective deficit in the acquisition of an eight-pair concurrent discrimination task (Experiment 2a). These lesions did not affect choice latency, magazine latency, or percentage of bias and did not have effects on other tasks in the current research, suggesting that the impaired acquisition of the anterior cingulate cortex-lesioned animals on this task cannot be attributed to motivational or motoric factors or to the tendency for animals to develop side preferences.

This task, when administered using "junk-object" stimuli (Zola-Morgan \& Squire, 1985) or items presented on slides (Aggleton, Nicol, Huston, \& Fairbairn, 1988; E. A. Gaffan, Aggleton, Gaffan, \& Shaw, 1990), is considered to be a sensitive test of amnesia in humans. Monkeys with lesions within the temporal lobe also are impaired on this task (Zola-Morgan \& Squire, 1985). These authors suggested that concurrent discrimination learning tasks are sensitive to amnesia because of a high mnemonic load for stimulusreward associations, thus requiring "declarative" memory (Cohen \& Squire, 1980) for their solution. It is argued that people with amnesia are unimpaired on difficult one-pair pattern discriminations because of a higher premium on the perceptual discrimination requirements, relative to stimulusreward learning demands, of this task (Zola-Morgan \& Squire, 1985). Anterior cingulate cortex-lesioned animals in this research exhibited this same pattern, suggesting that these animals may be deficient in their ability to acquire and remember multiple stimulus-reward associations. Damage to several other brain regions has been shown to impair concurrent discrimination learning; these regions include the rhinal cortex, the amygdala, and the mediodorsal thalamus (Hunt, Neave, Shaw, \& Aggleton, 1994; Mumby, Pinel, Kornecook, Shen, \& Redila, 1995; Rothblat, Vnek, Gleason, \& Kromer, 1993). These areas are all connected with the anterior cingulate cortex (Finch, 1993; Sripanidkulchai et al., 1984; Uylings \& van Eden, 1990) and thus may make up part of a system involved in the acquisition of concurrent discrimination tasks or in stimulus-reward learning in general (e.g., D. Gaffan, Murray, \& Fabre-Thorpe, 1993).

Zola-Morgan and Squire (1985) have argued that whereas people with amnesia are impaired on tests of "declarative" memory such as the eight-pair concurrent discrimination task, they are unimpaired on tasks requiring S-R "habit," or "procedural," memory. Similarly, the anterior cingulate cortex-lesioned animals in this research were unimpaired in 
the acquisition of a visuospatial conditional discrimination task that required the acquisition of procedural knowledge of what response to perform in the presence of a given stimulus (Experiment 1a). Information about the reinforcer, although clearly important as a training signal, did not provide information that could have been used to solve the task. Furthermore, in a version of this task carried out in an operant chamber, anterior cingulate cortex lesions facilitated acquisition (Bussey et al., 1996), and cholinergic denervation of the cingulate cortex had similar effects (Muir, Bussey, Everitt, \& Robbins, 1996). To account for these results, we have speculated that the anterior cingulate cortex may be involved in the acquisition of stimulus-reward associations and that stimulus-reward and S-R learning may, under certain circumstances, compete (Bussey et al., 1996; Muir, Bussey, Everitt, \& Robbins, 1996).

According to this account, when the stimulus-reward learning system is compromised, competition with the procedural S-R learning system is attenuated, resulting in facilitated S-R learning. This account, however, would predict that anterior cingulate cortex lesions should have facilitated acquisition of the conditional task in our research, but they did not. One possible explanation for this result is that for stimulus-reward learning to compete effectively with S-R learning, the stimulus-reward learning system must be adequately engaged during task acquisition. The conditions required for this to occur may not have been optimal in the touchscreen conditional task, whereas they were more so in the version of the task carried out in an operant chamber. Specifically, it is well established that the spatial and temporal contiguity of stimulus and reinforcer are important for stimulus-reinforcer learning (e.g., Rescorla \& Cunningham, 1979), and the discriminative stimuli and the reinforcer were much closer together spatially and temporally in the operant chamber, where they were presented on the same wall of the testing chamber, than in the current research, where the reward was delivered at the opposite end of the testing chamber. This possibility could be tested by manipulating the spatial and temporal contiguity of stimulus and reward in our apparatus.

Despite the focus of this discussion on the stimulusreward learning component of the eight-pair concurrent discrimination task, it is clear that this task and the one-pair discrimination task differ in more respects than merely their relative stimulus-reward learning demands. For example, interitem interference is much higher in the eight-pair task, and the average interstimulus interval (ISI) - the time elapsed between successive presentations of a given pair of stimuli-is much longer in the eight-pair task than in the one-pair task; indeed, it is on average at least eight times as long. Lesion effects could be related to these aspects of the tasks. Recently, however, anterior cingulate cortex lesions have been found to impair discrimination between a rewarded and a nonrewarded stimulus in a Pavlovian autoshaping task using only two easily discriminable stimuli and an average programmed ISI of $40 \mathrm{~s}$ (Bussey, Everitt, \& Robbins, 1997). This provides evidence that the stimulusreward learning demands, rather than factors related to interference or long ISIs, may have been responsible for the impairments observed in both this and the eight-pair concurrent discrimination task.

Further evidence for a role of the anterior cingulate cortex in stimulus-reward learning is that rats with lesions to this area were slower to collect reward than sham-lesioned animals or animals with lesions of the posterior cingulate cortex in both Experiments 1a and 4a. This may have been because these animals had incompletely acquired an association between the secondary reinforcers (i.e., tone and magazine light) and reward during pretraining. Consistent with this suggestion is the observation that this effect was observed only in tasks immediately following pretraining. However, because this effect did not reach significance in one case (Experiment 3), this interpretation must remain speculative.

\section{Effects of Posterior Cingulate Cortex Lesions}

Posterior cingulate cortex-lesioned animals were impaired during the late stages of learning a visuospatial conditional discrimination task (Experiment 1a). All these animals were able eventually to attain high levels of performance, suggesting that they had not reached a lesioninduced performance ceiling produced by factors such as attentional dysfunction. Furthermore, posterior cingulate cortex lesions disrupted only the conditional task and no other task in the current research; indeed, these animals demonstrated enhanced acquisition of the eight-pair concurrent discrimination task (Experiment 2a). These findings cast doubt on an attentional explanation for the results, unless the conditional task includes attentional requirements not present in these other tasks. Furthermore, this result replicates the findings of a previous study in which a visuospatial conditional task was conducted in an operant chamber, in which the discriminative stimuli were slow and fast flashing lights, the responses were left and right leverpresses, the reinforcer was a sucrose solution, and the task took many more sessions to acquire than the touchscreen version of the task used in the current research (Bussey et al., 1996).

Animals with lesions of the posterior cingulate cortex were selectively impaired during the late stages of learning (progressing from $70 \%$ to $85 \%$ ) in both the current research and that of Bussey et al. (1996), suggesting that the effects were not caused by factors related to the nature of the stimuli, responses, system of reinforcement, or general task difficulty. The associative demands of these two versions of the task are, however, identical; in both, the animal must learn a conditional rule of the type, "If A, go left and if B, go right." We have suggested that the posterior cingulate cortex may have a role in this type of S-R habit learning (Bussey et al., 1996). The posterior cingulate cortex receives projections directly from the visual cortex (area 18; Vogt, 1985) and projects strongly to the mediodorsal caudate nucleus (Domesick, 1969; McGeorge \& Faull, 1989), placing the posterior cingulate cortex in an ideal position in which to integrate visual information and response output.

An alternative hypothesis is that the deficit is related to the spatial requirements of the conditional task: right and left 
nose pokes in the current research and right and left leverpresses in the study of Bussey et al. (1996). Indeed, the suggestion has been made, based chiefly on the observation that posterior cingulate cortex lesions impair performance in the Morris water maze task (R. J. Sutherland et al., 1988), that the posterior cingulate cortex has a role in the processing of spatial information (R. J. Sutherland \& Hoesig, 1993). Although the water maze task requires the use of "allocentric" spatial information, and these conditional tasks more likely require the use of "egocentric" spatial information, this does not rule out the possibility that spatial requirements may have contributed to the deficit. However, Neave, Lloyd, Sahgal, and Aggleton (1994) have shown that excitotoxic lesions of the posterior cingulate cortex do not impair the acquisition or performance of a variety of egocentric and allocentric spatial tasks. Although it is still plausible that posterior cingulate cortex lesions are sensitive to certain classes of spatial problems or certain aspects of the water maze task, it is unlikely that, in light of the results of the study by Neave et al. (1994), the spatial requirements of the visuospatial conditional task are responsible for the impairment observed in the current research.

The posterior cingulate cortex may be necessary for normal conditional or S-R learning. However, the specific role this region would play in this form of learning is unclear. One possibility is that the posterior cingulate cortex is involved in response generation. Evidence for this proposal comes from unit-recording studies in behaving monkeys (Carlson \& Goldman-Rakic, 1995; Olson, Musil, \& Goldberg, 1993) and cats (Musil \& Olson, 1993). Those authors, using visual and memory guided eye and limb movement tasks, reported that neurons in the posterior cingulate cortex fired during the behavioral response but rarely before, suggesting that these neurons may be involved in the preparation and execution of movements. It is conceivable that the contribution of the posterior cingulate cortex becomes the most important during the late stages of learning, when there is a high premium on the ability to make efficient and correct responses to the target, be it a lever in an operant chamber or a response area on a computer screen.

A second effect obtained after lesions of the posterior cingulate cortex in the current research was that lesions of this region of cortex facilitated acquisition of the eight-pair concurrent discrimination task (Experiment 2a). Although this effect was relatively small, its significance is in complementing the deficits obtained after anterior cingulate cortex lesions on this task and in adding to the double dissociation between the two lesions on the conditional discrimination and eight-pair concurrent discrimination learning tasks. Furthermore, this result once again supports the notion of competing systems; however, the precise nature of this competition is unclear. One possibility, following the suggestions offered earlier that posterior cingulate cortex lesions impair habit learning or spatial discriminations, is that animals with posterior cingulate cortex lesions have less of a tendency to develop spatial response habits (i.e., side preferences), which normally can interfere with discrimination learning. However, the analysis of the percentage of bias measure revealed that posterior cingulate cortexlesioned animals were no less or more side biased than sham-lesioned or anterior cingulate cortex-lesioned animals in any of our experiments.

\section{Effects of Medial Frontal Cortex Lesions}

Lesions of the medial frontal cortex produced a profound and robust impairment in reversal learning, but only when stimuli were difficult to discriminate (Experiment 3 ). Further analysis of the data in terms of errors required to attain a criterion of $39 \%$ correct choices, compared with errors committed between $39 \%$ and $85 \%$, revealed that this impairment was caused by the animals with medial frontal cortex lesions committing considerably more errors than shamoperated animals when progressing between the criteria of $39 \%$ and $85 \%$. Jones and Mishkin (1972) suggested that such a pattern of results may be indicative of a deficit in stimulus-reward learning. However, Bussey et al. (1997) failed to observe deficits after medial frontal cortex lesions in an autoshaping task in which animals learned to approach the stimuli predictive of reward. Furthermore, medial frontal cortex-lesioned animals were unimpaired in the acquisition of eight stimulus-reward associations in the eight-pair concurrent discrimination task in Experiment 2b. Particularly problematic for a stimulus-reward learning account of this pattern of results is the observation that medial frontal cortex lesions had no effect on reversal learning when the stimuli were easily discriminable (Experiment $\mathbf{4 b}$ ). If anything, this manipulation-which served to decrease the perceptual discrimination aspects of the task, thereby increasing the premium on the stimulus-reward learning demands of the task-should have produced a greater deficit in animals with impairments in stimulus-reward learning. The observation that the deficit exhibited by medial frontal cortex-lesioned animals depends on the discriminability of the stimuli is more suggestive of an impairment in the ability of the rats to attend to the stimulus features according to which stimuli must be discriminated. In this respect, medial frontal cortex-lesioned rats might be regarded as the converse of overtrained rats, whose attention can become so well "tuned" to the relevant stimulus attributes in a discrimination problem that when the stimulus-reward contingencies are reversed, these animals actually reverse more rapidly than nonovertrained rats (the "overtraining reversal effect"; Reid, 1953). Intriguingly, the facilitation caused by overtraining in these cases is observed late in reversal learning and only when stimuli are difficult to discriminate (N. S. Sutherland \& Mackintosh, 1971). Evidence from other studies supports a role for the medial cortex of the rat in attentional processes. For example, Muir, Everitt, and Robbins (1996) reported that medial frontal cortex lesions in the rat impair a continuous performance test of visual attention (the "five-choice serial reaction time task"). Furthermore, prominent accounts of human prefrontal function feature a role for this region in aspects of attention (Knight \& Grabowecky, 1995; Shallice, 1988). It remains to be determined whether attentional factors contribute to spatial reversal learning deficits in rats with prefrontal cortex 
lesions (Divac, 1971) or whether such deficits are attributable to the spatial nature of the tasks. This is an important issue in evaluating the utility of the rat prefrontal cortex as a model for frontal cortical function in human and other primates (Preuss, 1995).

\section{Conclusions}

The results of our research provide evidence for the functional dissociability of the anterior cingulate, posterior cingulate, and medial frontal cortices of the rat and offer clues about what the precise functions of these brain regions might be. Specifically, the anterior cingulate cortex appears to have a role in appetitive Pavlovian conditioning. The posterior cingulate cortex is involved in the acquisition of visuospatial conditional tasks, which may reflect a general involvement of this region in stimulus-response learning, perhaps in the capacity of response generation. The medial frontal cortex does not appear to be involved in basic learning processes but instead may have a "supervisory" role in maintaining attention to relevant stimulus features during learning. These suggestions are consistent with a theoretical framework that regards the brain as consisting of anatomically distinct systems that mediate dissociable forms of learning, memory, and attention.

\section{References}

Aggleton, J. P., Nicol, R. M., Huston, A. E., \& Fairbairn, A. F. (1988). The performance of amnesic subjects on tests of experimental amnesia in animals: Delayed matching-to-sample and concurrent learning. Neuropsychologia, 26, 265-272.

Becker, J. T., \& Olton, D. S. (1980). Object discrimination by rats: The role of frontal and hippocampal systems in retention and reversal. Physiology \& Behavior, 24, 33-38.

Brog, J. S., Salyapongse, A., Deutch, A. Y., \& Zahm, D. S. (1993). The patterns of afferent innervation of the core and shell in the "accumbens" part of the rat ventral striatum: Immunohistochemical detection of retrogradedly transported fluoro-gold. Journal of Comparative Neurology, 338, 255-278.

Bussey, T. J., Everitt, B. J., \& Robbins, T. W. (1997). Dissociable effects of cingulate and medial frontal cortex lesions on stimulusreward learning using a novel Pavlovian autoshaping procedure for the rat: Implications for the neurobiology of emotion. Behavioral Neuroscience, 111, 908-919.

Bussey, T. J., Muir, J. L., Everitt, B. J., \& Robbins, T. W. (1996). Dissociable effects of anterior and posterior cingulate cortex lesions on the acquisition of a conditional visual discrimination: Facilitation of early learning vs. impairment of late learning. Behavioural Brain Research, 82, 45-56.

Bussey, T. J., Muir, J. L., \& Robbins, T. W. (1994). A novel automated touchscreen procedure for assessing learning in the rat using computer graphic stimuli. Neuroscience Research Communications, 15, 103-110.

Carlson, S., \& Goldman-Rakic, P. (1995). Dissociation of movement related neuronal activity in posterior cingulate cortex in monkeys performing oculomotor and manual delayed response tasks. Society for Neuroscience Abstracts, 21, 1929.

Cohen, N. J., \& Squire, L. R. (1980). Preserved learning and retention of pattern-analyzing skill in amnesia: Dissociation of knowing how and knowing that. Science, 210, 207-210.
Devinsky, O., Morrell, M. J., \& Vogt, V. A. (1995). Contributions of anterior cingulate cortex to behavior. Brain, 118, 279-306.

Dias, R., Robbins, T. W., \& Roberts, A. C. (1996). Dissociation in prefrontal cortex of affective and attentional shifts. Nature, 380 , 69-72.

Divac, I. (1971). Frontal lobe system and spatial reversal in the rat. Neuropsychologia, 9, 175-183.

Divac, I., \& Diemer, N. H. (1980). Prefrontal system in the rat visualized by means of labeled deoxyglucose: Further evidence for functional heterogeneity of the neostriatum. Journal of Comparative Neurology, 190, 1-13.

Domesick, V. B. (1969). Projections from the cingulate cortex in the rat. Brain Research, 12, 296-320.

Dudchenko, P., \& Sarter, M. (1991). GABAergic control of basal forebrain cholinergic neurons and memory. Behavioural Brain Research, 42, 33-42.

Finch, D. M. (1993). Hippocampal, subicular, and entorhinal afferents and synaptic integration in rodent cingulate cortex. In B. A. Vogt \& M. Gabriel (Eds.), Neurobiology of cingulate cortex and limbic thalamus: A comprehensive handbook (pp. 224-248). Boston: Birkhauser.

Gabriel, M. (1993). Discriminative avoidance learning: A model system. In B. A. Vogt \& M. Gabriel (Eds.), Neurobiology of cingulate cortex and limbic thalamus: A comprehensive handbook (pp. 478-523). Boston: Birkhauser.

Gaffan, D., Murray, E. A., \& Fabre-Thorpe, M. (1993). Interaction of the amygdala with the frontal lobe in reward memory. European Journal of Neuroscience, 5, 968-975.

Gaffan, E. A., Aggleton, J. P., Gaffan, D., \& Shaw, C. (1990). Concurrent and sequential pattern discrimination learning by patients with Korsakoff amnesia. Cortex, 26, 381-397.

Groenewegen, H. J., Berendse, H. W., Wolters, J. G., \& Lohman, A. H. M. (1990). The anatomical relationship of the prefrontal cortex with the striatopallidal system, the thalamus, and the amygdala: Evidence for a parallel organization. Progress in Brain Research, 85, 95-118.

Howell, D. C. (1987). Statistical methods for psychology (2nd ed.). Boston: PWS.

Hunt, P. R., Neave, N., Shaw, C., \& Aggleton, J. P. (1994). The effects of lesions to the fornix and dorsomedial thalamus on concurrent discrimination learning by rats. Behavioural Brain Research, 62, 195-205.

Jones, B., \& Mishkin, M. (1972). Limbic lesions and the problem of stimulus-reinforcer associations. Experimental Neurology, 36, 362-377.

Knight, R. T., \& Grabowecky, M. (1995). Escape from linear time: Prefrontal cortex and conscious experience. In M. S. Gazzaniga (Ed.), The cognitive neurosciences (pp. 1357-1371). Cambridge, MA: Bradford.

Kolb, B. (1984). Functions of the frontal cortex of the rat: A comparative review. Brain Research Reviews, 8, 65-98.

Kolb, B. (1990). Animals models for human PFC-related disorders. Progress in Brain Research, 85, 501-519.

Lopez da Silva, F. H., Witter, M. P., Boeijinga, P. H., \& Lohman, A. H. M. (1990). Anatomic organization and physiology of the limbic cortex. Psychological Review, 70, 453-511.

McGeorge, A. J., \& Faull, R. L. M. (1989). The organization of the projection from the cerebral cortex to the striatum in the rat. Neuroscience, 29, 503-537.

Meunier, M., Jaffard, R., \& Destrade, C. (1991). Differential involvement of anterior and posterior cingulate cortices in spatial discriminative learning in a T-maze in mice. Behavioural Brain Research, 44, 133-143.

Muir, J. L., Bussey, T. J., Everitt, B. J., \& Robbins, T. W. (1996). Dissociable effects of AMPA-induced lesions of the vertical limb 
of the diagonal band of Broca on performance of the 5-choice serial reaction time task and on acquisition of a conditional visual discrimination. Behavioural Brain Research, 82, 31-44.

Muir, J. L., Everitt, B. J., \& Robbins, T. W. (1996). The cerebral cortex of the rat and visual attentional function: Dissociable effects of mediofrontal, cingulate, anterior dorsolateral, and parietal cortex lesions on a five-choice serial reaction time task. Cerebral Cortex, 6, 470-481.

Mumby, D. G., Pinel, J. P. J., Kornecook, T. J., Shen, M. J., \& Redila, V. A. (1995). Memory deficits following lesions of hippocampus or amygdala in rat: Assessment by an objectmemory test battery. Psychobiology, 23, 26-36.

Musil, S. Y., \& Olson, C. R. (1993). The role of cat cingulate cortex in sensorimotor integration. In B. A. Vogt \& M. Gabriel (Eds.), Neurobiology of cingulate cortex and limbic thalamus: $A$ comprehensive handbook (pp. 345-365). Boston: Birkhauser.

Neave, N., Lloyd, S., Sahgal, A., \& Aggleton, J. P. (1994). Lack of effect of lesions in the anterior cingulate cortex and retrosplenial cortex on certain tests of spatial memory in the rat. Behavioural Brain Research, 65, 89-101.

Neave, N., Nagle, S., Sahgal, A., \& Aggleton, J. P. (1996). The effects of discrete cingulum bundle lesions in the rat on the acquisition and performance of two tests of spatial working memory. Behavioural Brain Research, 80, 75-85.

Olson, C. R., Musil, S. Y., \& Goldberg, M. E. (1993). Posterior cingulate cortex and visuospatial cognition: Properties of single neurons in the behaving monkey. In B. A. Vogt \& M. Gabriel (Eds.), Neurobiology of cingulate cortex and limbic thalamus: $A$ comprehensive handbook (pp. 366-380). Boston: Birkhauser.

Paxinos, G., \& Watson, C. (1986). The rat brain in stereotaxic coordinates (2nd ed.). New York: Academic Press.

Preuss, T. M. (1995). Do rats have a prefrontal cortex? The Rose-Woolsey-Akert program reconsidered. Journal of Cognitive Neuroscience, 7, 1-24.

Ray, J. P., \& Price, J. L. (1992). The organization of the thalamocortical connections of the mediodorsal thalamic nucleus in the rat, related to the ventral forebrain-prefrontal cortex topography. Journal of Comparative Neurology, 323, 167-197.

Reid, L. S. (1953). The development of noncontinuity behavior through continuity learning. Journal of Experimental Psychology, 46, 107-112.

Rescorla, R. A., \& Cunningham, C. L. (1979). Spatial contiguity facilitates Pavlovian second-order conditioning. Journal of Experimental Psychology: Animal Behavior Processes, 5, 152161.

Rothblat, L. A., Vnek, N., Gleason, T. C., \& Kromer, L. F. (1993). Role of the parahippocampal region in spatial and non-spatial memory: Effects of parahippocampal lesions on rewarded alternation and concurrent discrimination learning in the rat. Behavioural Brain Research, 55, 93-100.

Sesack, S. R., Deutch, A. Y., Roth, R. H., \& Bunney, B. S. (1989). Topographical organization of the efferent projections of the medial prefrontal cortex in the rat: An anterograde tract-tracing study with phaseolus vulgaris leucoagglutinin. Journal of Comparative Neurology, 290, 213-242.

Shallice, T. (1988). From neuropsychology to mental structure. New York: Cambridge University Press.

Sripanidkulchai, K., Sripanidkulchai, B., \& Wyss, J. M. (1984). The cortical projection of the basolateral nucleus of the rat: A retrograde fluorescent dye study. Journal of Comparative Neurology, 229, 419-431.

Sutherland, N. S., \& Mackintosh, N. J. (1971). Mechanisms of animal discrimination learning. New York: Academic Press.

Sutherland, R. J., \& Hoesig, J. M. (1993). Posterior cingulate cortex and spatial memory: A microlimnology analysis. In B. A. Vogt \& M. Gabriel (Eds.), Neurobiology of cingulate cortex and limbic thalamus: A comprehensive handbook (pp. 461-477). Boston: Birkhauser.

Sutherland, R. J., Whishaw, I. Q., \& Kolb, B. (1988). Contributions of cingulate cortex to two forms of spatial learning and memory. Journal of Neuroscience, 8, 1863-1872.

Swanson, L. W. (1992). Brain maps: Structure of the rat brain (1st ed.). Amsterdam: Elsevier.

Uylings, H. B. M., \& van Eden, C. G. (1990). Qualitative and quantitative comparison of the prefrontal cortex in rat and in primates, including humans. Progress in Brain Research, 85, $31-62$.

Vogt, B. A. (1985). Cingulate cortex. In A. Peters \& E. G. Jones (Eds.), Cerebral cortex: Vol. 4. Association and auditory cortices (pp. 89-149). New York: Plenum.

Vogt, B. A., Finch, D. M., \& Olson, C. R. (1992). Functional heterogeneity in cingulate cortex: The anterior executive and posterior evaluative regions. Cerebral Cortex, 2, 435-443.

Zilles, K. (1985). The cortex of the rat: A stereotaxic atlas (1st ed.). Berlin: Springer-Verlag.

Zola-Morgan, S., \& Squire, L. R. (1985). Medial temporal lesions in monkeys impair memory on a variety of tasks sensitive to human amnesia. Behavioral Neuroscience, 89, 22-34.

Received September 17, 1996

Revision received March 10, 1997

Accepted April 22, 1997 